\title{
Remarks on lattice gauge theories with infrared-attractive fixed points
}

\author{
Thomas DeGrand, Anna Hasenfratz \\ Department of Physics, University of Colorado, Boulder, CO 80309, USA \\ Abstract
}

Theories of interacting gauge fields and fermions can possess a running gauge coupling with an infrared attractive fixed point (IRFP). We present a minimal description of the physics of these systems and comment on some simple expectations for results from lattice simulations done within the basin of attraction of the IRFP in these theories. 


\section{INTRODUCTION}

Interest in non-Abelian lattice gauge theories which differ from QCD in having more quark flavors or quarks in larger representations has been growing in recent years. These studies aim to address physics that may appear at the energy of the Large Hadron Collider or beyond, described by theoretical constructs such as technicolor [1] or "unparticles" [2].

Most of the interesting theories are asymptotically free and an infinite cut-off limit can be defined at the ultraviolet Gaussian fixed point (FP) at bare couplings $g^{2}=0, m=$ 0 . Starting with bare parameters near the FP both the running gauge coupling and the mass increase as the energy scale is lowered. As long as the coupling $g^{2}$ remains small the perturbative renormalization group $\beta$ function describes its running. There are two possibilities at stronger coupling[3, 4]. The first is when the Gaussian fixed point is the only fixed point with a diverging correlation length. A familiar example is QCD with two light flavors. The $\beta$ function stays negative even at strong coupling, chiral symmetry is spontaneously broken and the model is confining. The second possibility is that the $\beta$ function develops another zero, corresponding to an infrared attractive fixed point (IRFP). In this case the infrared physics of the massless theory will possess a conformal symmetry that precludes confinement and the spontaneous breaking of chiral symmetry. These are the "unparticle" theories. Just before the conformal window opens up there is a possibility that the $\beta$ function, while staying negative, becomes small, thus the running of the coupling slows down to "walking". Such theories are leading candidates for technicolor models.

Most of the interesting cases in the literature have nonperturbative dynamics. The theoretical approaches used to analyze their behavior are not under complete control. Accordingly, several groups have begun to use lattice methods to investigate these models. In at least three cases (an $S U(3)$ gauge group with 12 flavors of fundamental representation fermions [5, 6], $S U(3)$ with two flavors of symmetric-representation fermions [7], and $S U(2)$ with two flavors of adjoint representation fermions [8]) authors have reported evidence for an IRFP theory.

This short note is written to present the simplest theoretical description of IRFP theories. While we believe that a considerable fraction of the lattice community is familiar with the physics we will describe, we have participated in enough conversations and read enough published papers in which the description we will give was incompletely presented, that we feel a review might be useful. A large part of the non-lattice literature about IRFP theories is devoted to describing the transition from an IRFP system to a confining one, as the number of flavors of fermions is reduced [9, 10, 11, 12, 13]. Our "theoretical minimum" might be useful as a benchmark against which simulation results and these predictions can be compared.

After some introductory remarks, we would like to make two points:

- When a theory is in the basin of attraction of an IRFP, its gauge coupling is irrelevant. The only relevant coupling, which controls the leading scaling behavior of the theory, is the fermion mass.

- The value of the IRFP gauge coupling, $g^{*}$, is scheme-dependent. Spectral observables cannot depend on its value, and can only be sensitive to the extreme values of the bare gauge coupling which mark the basin of attraction of the IRFP region. The renormalization group flow is always towards $g^{*}$, but its absolute direction depends on the scheme-dependent location of $g^{*}$. 
We now elaborate on these points and their consequences.

\section{MAPPING THE PHASE STRUCTURE WITH THE RENORMALIZATION GROUP}

The renormalization group $(\mathrm{RG})$ transformation is a frequently used tool in studying the infinite cut-off limit of quantum field theories. There are two different approaches. The Callan-Symanzik equation describes the problem from the perturbative point of view and is most often used in connection with continuum regularization schemes. It describes the change of the parameters of the theory as the function of the cut-off, or equivalently, the renormalization subtraction mass $\mu$. The calculation is perturbative and only parameters already present in the Lagrangian are considered. For example the $\beta$ function

$$
\beta\left(g^{2}\right)=\frac{d g^{2}}{d \log \left(\mu^{2}\right)}
$$

describes the running of the coupling $g^{2}$, and there are similar expressions for the mass and any other couplings present. Zeros of the $\beta$ function correspond to either ultraviolet or infrared fixed points (UVFP and IRFP), depending on the slope of $\beta\left(g^{2}\right)$ (negative or positive, respectively).

The other option is the inherently non-perturbative Wilson RG approach. There one considers the evolution of all the possible couplings of the system under an RG transformation that preserves the internal symmetries of the system but integrates out the cut-off level UV modes. The fixed points of the transformation are characterized by the number of relevant couplings or operators, i.e. couplings that flow away from the FP. Continuum (or infinite cut-off) limits are defined when the relevant couplings are tuned towards the FP. Irrelevant couplings flow in the IR to values which are independent of their UV values. The number of relevant operators and their speed along the RG flow lines are universal properties, related to the critical properties of the underlying continuum limit. However the location of the FP is not physical, in fact different RG transformations have different fixed points.

The relation between the Callan-Symanzik and Wilson RG approaches are straightforward: the Callan-Symanzik $\beta$ function describes the $\mathrm{RG}$ flow of one or a few couplings, usually implicitly in perturbation theory. The zeroes of the $\beta$ function correspond to the fixed points of the Wilson RG. While the Callan-Symanzik equations can provide the first hint about the phase structure, unless the predictions are well controlled by perturbation theory, a non-perturbative approach is needed to verify the existence and study the properties of the relevant fixed points. Lattice calculations provide an excellent tool for that. We believe that the Wilson RG approach is better suited to interpret lattice results and in the following we will use mainly that language.

\section{THE PHASE DIAGRAM OF GAUGE THEORIES WITH MANY FERMIONS}

Consider a theory with $S U\left(N_{c}\right)$ gauge group, coupled to $N_{f}$ flavors of fermions in representation $R$. In our mind is a lattice simulation and that is the phase diagram we will describe. The Gaussian fixed point at $g^{2}=0, m=0$ is well understood perturbatively. The mass is a relevant operator and it will presumably will remain so even at strong gauge coupling. $m=0$ is a critical surface and we want to investigate the running of the gauge 
coupling along it. In the perturbative region this is described by the $\beta$ function which is universal up to two loop level:

$$
\begin{gathered}
\beta\left(g^{2}\right)=\frac{d g^{2}}{d \log \left(\mu^{2}\right)}=\frac{b_{1}}{16 \pi^{2}} g^{4}+\frac{b_{2}}{\left(16 \pi^{2}\right)^{2}} g^{6}+\ldots \\
b_{1}=-\frac{11}{3} N_{c}+\frac{4}{3} N_{f} T(R) \\
b_{2}=-\frac{34}{3} N_{c}^{2}+N_{f} T(R)\left(\frac{20}{3} N_{c}+4 C_{2}(R)\right) .
\end{gathered}
$$

If the fermion number $N_{f} \leq(11 / 4) N_{c} / T(R)$ (16.5 for fundamental representation fermions in QCD), $b_{1}<0$ and both the gauge coupling and the mass are relevant operators at the Gaussian FP. The theory is asymptotically free, the coupling $g^{2}$ increases as the lattice spacing (or inverse cut-off) increases. For small number of fermions the perturbative $\beta$ function remains negative even at strong coupling. Lattice simulations with $N_{f}=2,2+1$, and to some extent up to $N_{f} \leq 8$ verify this: QCD with 2-8 light fermions is confining and chirally broken everywhere [14]. With increasing $N_{f}$ the two-loop term in Eq. 2 changes sign, suggesting a possible zero in $\beta(g)$. When the quarks are in higher representations of the gauge group, this happens more easily. The zero of $\beta(g)$ corresponds to an IRFP in the gauge coupling. That is, the gauge coupling is irrelevant, and the only relevant parameter is the mass term. The continuum limit is defined in the basin of attraction of this IRFP by tuning the mass to zero.

The existence of the perturbatively predicted IRFP and the properties of the theory at even stronger coupling have to be studied with non-perturbative methods, like lattice simulations. The lattice provides an UV regularization scheme which is equivalent to continuum schemes at weak coupling. At strong gauge coupling lattice artifacts can completely change the system. It is generally believed that in the strong coupling limit lattice models are always confining and chirally broken, independent of $N_{f}$ [15, 16, 17], This was observed in early $N_{f}=16$ simulations [18, 19], though numerical results in Ref. [20] contradict this. In any case, the details in the strong coupling region are not universal and, for now, not very important either. Fig. 1 shows a conjectured phase diagram in the $g^{2}$ vs $m$ plane when an IRFP exists. The thick line at large $g^{2}$ shows the (possible) confining and chirally broken phase, but around the IRFP the theory is chirally symmetric and deconfined. The dashed line indicates the phase boundary between the two phases. It is likely only a crossover at finite mass. We note that in a recent paper it was suggested that in these many-fermion theories with an IRFP there is always another FP (zero of the $\beta$ function) at a stronger coupling [13]. If this conjecture is valid, the phase diagram has another UVFP at $m=0$. This could be the boundary between the conformal and strong coupling phases in Fig. 11, or an entirely new critical point.

It is important to note that while at a UVFP an observable phase transition occurs, there is nothing observable at the IRFP. The location of an IRFP on the critical surface is not physical. It depends on the specific renormalization group transformation, and therefore no physical observable can identify it. In particular, this means that studies of candidate theories which only measure spectroscopic observables cannot directly detect coupling constant flow through the dependence of observables on irrelevant bare couplings. They can only detect the perimeter of the basin of attraction of the FP. 


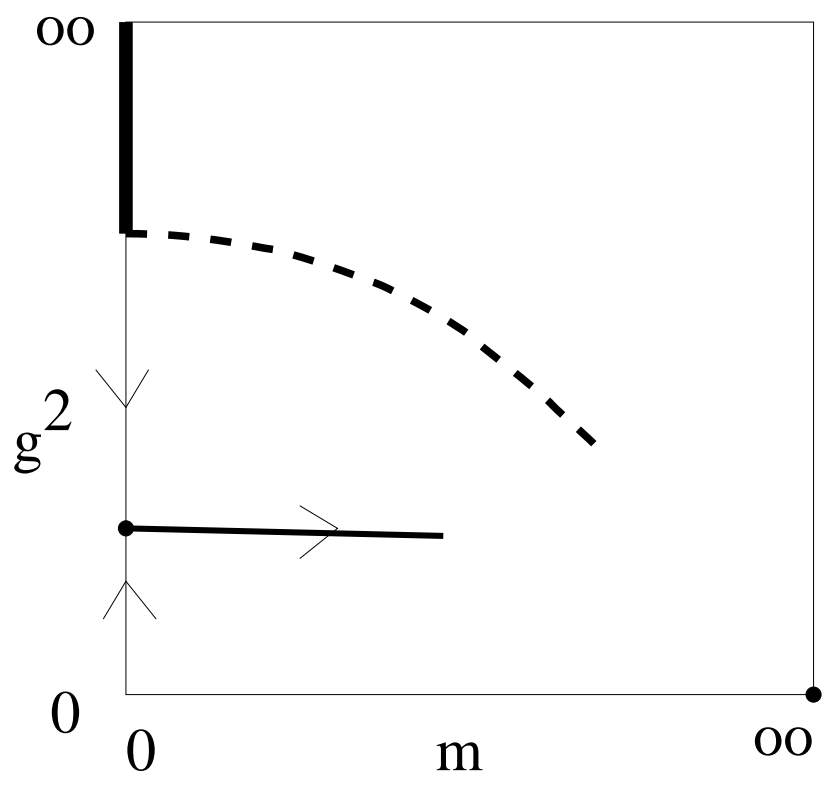

FIG. 1: A conjectured phase diagram for a lattice theory with an IRFP. The thick line at $m=0$ is the confining, strong-coupling region of the massless theory. The dotted line separates a confining phase (which includes the entire $g^{2}$ line at large fermion mass) from the basin of attraction of the IRFP. Arrows show some IR flow lines and the renormalized trajectory extending from the critical point.

\section{ANALYSIS OF THE PHASE DIAGRAM}

To begin, note first that the gauge coupling plays a very different role around the UVFP and in the vicinity of the IRFP or trivial phases: at the UVFP $g$ is relevant, and to drive the correlation length(s) to infinity in units of the cutoff scale involves a fine tuning of $g$ toward zero. (To be precise, the gauge coupling is marginally relevant with respect to the Gaussian fixed point, because $\beta\left(g^{2}\right) \sim b_{1} g^{4}$.) In the other phases, IR physics is independent of the value of $g$ at the UV scale, because the IR flow of the gauge coupling is into its fixed point value: the gauge coupling is irrelevant. This is a simple example illustrating the general feature, that relevance or irrelevance of an operator is measured with respect to a fixed point. The presence of the IRFP means that the linearized behavior of the beta function near the fixed point is

$$
\frac{d g^{2}}{d s}=y_{g}\left(g^{2}-g^{2 *}\right)
$$

where $s$ is the (IR) scale change, $\mu \rightarrow e^{-s} \mu$, and $y_{g}<0$ is the scaling exponent of the gauge coupling. The running coupling then is $g^{2}(s)=g^{2 *}+\left(g_{0}^{2}-g^{2 *}\right) e^{y_{g} s}$, i.e. $g(s) \rightarrow g^{*}$ as $s \rightarrow \infty$. We discuss the value of $y_{g}$ below.

Consider next the general situation, a theory in $D$ Euclidean dimensions defined by a set of scaling operators $\{u\}$. Under a real-space blocking by a factor $b$, the operators run multiplicatively to/from their FP values, $u_{i}^{\prime}-u_{i 0}=b^{y_{i}}\left(u_{i}-u_{i 0}\right)$, with the usual assignment of relevancy, irrelevancy, or marginality, depending on whether $y_{i}$ is positive, negative, or zero. Let us assume that the mass is the only relevant coupling and its FP value is zero; its exponent we will label as $y_{m}$. Only the leading exponent governs the correlation length $\xi$, 
which diverges with the usual algebraic behavior

$$
\xi \sim m^{-\frac{1}{y_{m}}}
$$

A standard textbook analysis tells us that the the singular part of the free energy per site (it is the part of the free energy containing all the non-analyticity at the critical point) scales as

$$
f_{s}\left(m, u_{i}\right)=m^{D / y_{m}} f_{s}\left(m_{0}, u_{i 0}+\left(u_{i}-u_{i 0}\right)\left(\frac{m}{m_{0}}\right)^{\left|y_{i}\right| / y_{m}}\right),
$$

which can be Taylor expanded as

$$
f_{s}(m)=m^{D / y_{m}}\left(A_{1}+A_{2} m^{\left|y_{i}\right| / y_{m}}\right),
$$

where $A_{1}$ and $A_{2}$ are non-universal constants. All other observables have a similar expansion.

Precisely at $m=0$ the entire basin of attraction of the FP is critical - at long distance all correlation functions decay algebraically

$$
\left\langle\phi_{i}(r) \phi_{i}(0)\right\rangle=\sum_{j} \frac{E_{j}}{r^{2\left(D-y_{j}\right)}} .
$$

This behavior is only achieved by setting the quark mass to zero. The nonanalytic dependence of the free energy on the fermion mass is responsible for the algebraic behavior of correlation functions in the massless theory. Eq. 8 is only true asymptotically. At distances which are comparable to the UV cutoff (lattice spacing $a$ ) there are additional contributions. They arise from the non-singular part of the free energy, from physics at the intermediate scales which has been integrated out in the construction of Eq. 6. These give extra contributions going as $\exp (-r / a)$.

On the $m=0$ critical surface all couplings are irrelevant; they all flow into the IRFP. The interesting physical quantity near the FP is the leading relevant exponent. This is not accessible from a lattice-based RG study at $m=0$ (for example, a conventional Schrödinger functional study which computes $\beta(g))$. These studies only give $y_{g}$ because by construction they are only sensitive to flow into the FP. They tell us that there is an IRFP, no more.

Unitarity bounds for $D=4$ conformal field theories [21] constrain the scaling dimension of the condensate $\langle\bar{\psi} \psi\rangle \sim \mu^{\gamma}$ to lie in the range $3>\gamma>1$. Since $m \bar{\psi} \psi$ is scale invariant and since all dimensionful quantities scale with the correlation length as in Eq. 5, $\gamma=$ $4-y_{m}$. (There are other definitions for the scaling dimension in the literature but this one is consistent with the bound of Ref. [21].) We do not want to discuss specific techniques for determining it in a simulation, and defer a discussion of possible values for $y_{m}$ to later work [22]. In the meantime, however, there is one case for which it is easy to expose the operator scaling hierarchy [4, 23]. This is a lattice theory of $N_{c}$ colors and $N_{f}$ flavors in some representation, and that the limit $N_{c} \rightarrow \infty$ and $N_{f} / N_{c}$ fixed, so that the Banks-Zaks FP occurs at a small value of $g^{*} \sim \epsilon$. Engineering dimensions give basically everything. For this theory, the condensate has scaling exponent $\gamma=3-O(\epsilon)$. The gauge coupling contributes a scaling exponent $y_{g} \sim \epsilon$ (which can be computed in perturbation theory), and lattice-based artifacts contribute negative $y_{i}$ 's.

This is the upper end of the conformal window. What happens at the lower end of the conformal window, where the system converts from being an IRFP theory to a confining, chirally broken theory, is a subject of long-standing interest. Observations of $y_{m}$ will likely be needed to elucidate this point. 


\section{CONSEQUENCES FOR SIMULATIONS}

One feature of IRFP theories is concealed by the Callan-Symanzik formalism: the location of a fixed point is not universal. Different choices of renormalization group transformations will result in different values of $g^{*}$. This has several consequences for simulations.

First of all, observables taken from correlation functions cannot depend on the actual value of the FP coupling determined in some scheme: the correlation function does not know about the scheme. The important part of expressions like Eq. 7 is the statement that the size of scale violations in observables is $\left(\frac{m}{m_{0}}\right)^{\left|y_{i}\right| / y_{m}}$.

Real QCD (for example, an $S U(3)$ gauge theory with a small number of flavors of fundamental representation fermions) is more complicated than an IRFP theory. Now there are two relevant couplings which flow away from the $\left(g^{2}, m_{q}\right)=(0,0)$ fixed point. Lattice observables (like masses) depend strongly on both of these couplings. Indeed, making lattice predictions typically involves first finding a map of $m_{q}\left(g^{2}\right)$ along which some mass ratio is fixed, then taking the continuum limit by moving along this line toward the UVFP. If one tried to do this for an IRFP theory, one would be trying to express (or tune) the relevant coupling in terms of an irrelevant coupling.

Next, because different choices for renormalization schemes result in different values for $g^{*}$, it is easy to imagine a situation where the running coupling in one scheme is increasing under flow to the IR, while the running coupling in another scheme is decreasing. In fact, this is another diagnostic for the presence of an IRFP: one can vary the renormalization group transformation and see if the direction of coupling constant flow changes.

Much continuum beyond-Standard Model phenomenology uses a scale dependent coupling constant defined in some particular scheme, such as $\overline{M S}$. A lattice calculation of such a coupling involves both a lattice simulation and an additional scheme matching calculation. Critital exponents such as $y_{m}$ are scheme-independent.

Simulations are carried out at particular values of the bare couplings. Eq. 7 tells us that the influence of the gauge coupling on observables dies away in the zero mass limit. In a sense, varying the gauge coupling while remaining near the basin of attraction of the IRFP is like doing lattice simulations for ordinary QCD with several choices of the lattice action - in the scaling limit, the answer is not supposed to depend on the choice of action. Away from infinite correlation length, different lattice actions will give different predictions for observables. But these differences are just scale violations. In this case, the choice of bare gauge coupling is as much a choice of the action as a particular discretization would be.

The correlation length $\xi$ only varies with the bare mass according to Eq. 5 when the system size $L$ is much larger than $\xi$. This is probably the the most serious practical constraint on the choice of bare parameters.

The size of the exponent $y_{g}$ also acts to minimize the dependence of simulation results on $g^{2}$ : It is likely the value of $y_{g}$ is very small. The exponent can be seen in simulations. The authors of Ref. [6] report $y_{g} \sim-0.15$. The authors of Ref. [8] do not quote a number, but they give a plot of the $\beta$ function from which a value of about -0.16 can be inferred. The authors of Ref. [7] only show an integrated beta function, but parameterizing their data with a simple model (a second order formula with the perturbative $b_{1}$ and a $b_{2}$ chosen to give a zero) also gives a small $y_{g} \sim-0.06$. Gardi and Grunberg[12] present perturbative calculations for both QCD-like and supersymmetric theories. Only at the bottom of the conformal window, and only for non-supersymmetric theories, is $-y_{g}$ greater than about 0.5. If that is the case, then in formulas like Eq. 7 the correction term is nearly a constant. 
This has two consequences:

- In a lattice simulation of a particular system which is a candidate for being an IRFP theory, the observation that long distance observables become independent of $g$ at small $m$ is a positive indication of IRFP behavior

- In the basin of attraction of an IRFP, one does not need to fine tune the gauge coupling; one can compute wherever it is convenient.

What evidence is there for the behavior we have described, from published results for candidate IRFP theories? Two groups have studied the $S U(2)$ gauge group with two flavors of adjoint fermions. This model has a confining strong coupling phase and a weak coupling phase which the authors of Ref. [8] have argued is conformal. The authors of Ref. [24] measure the pseudoscalar and vector masses in finite volume at zero quark mass. Finite volume limits the correlation length to be proportional to the system size. They observe (in their Fig. 5) that these masses are independent of the bare gauge coupling in the weak coupling phase. They also note the constancy of Creutz ratios with respect to gauge coupling (ratios of Wilson loops) in this phase; see their Fig. 7. The authors of Ref. [25] observe that their pseudoscalar and vector masses are independent of gauge coupling in the weak coupling phase; compare their figures 5-8.

One of us collaborated with Svetitsky and Shamir in studies of $S U(3)$ gauge theory with two flavors of sextet fermions [26]. This system also has a weak coupling phase which is a candidate for an IRFP phase. It is easier to replot data published there: Fig. 2 shows the pseudoscalar mass as a function of the quark mass, from simulations performed at many values of the gauge coupling $\left(\beta=6 / g^{2}\right)$ in the weak coupling phase. The finite simulation volume prevents the vanishing of the pseudoscalar mass at zero quark mass. Results are similar to what is seen in the $S U(2)$, adjoint case, i.e., weak dependence of the meson mass on the gauge coupling.

\section{CONCLUSIONS}

We have presented a minimal scenario for physics within the basin of attraction of an IRFP. There is one relevant coupling, the fermion mass, which governs the correlation length through Eq. 5. All other couplings are irrelevant. This scenario gives a context for interpreting simulation results and has observable consequences. Probably, convincing evidence for an IRFP requires RG studies. However, the simple behavior we have described provides additional markers for the description of an IRFP theory with a single relevant operator.

\section{Acknowledgments}

We thank P. Damgaard, U. M. Heller, D. M. Kaplan, T. G. Kovacs, L. Del Debbio, B. Svetitsky and Y. Shamir for discussions. This work was supported in part by the US Department of Energy.

[1] For a review of the phenomenology, see C. T. Hill and E. H. Simmons, Phys. Rept. 381, 235 (2003) [Erratum-ibid. 390, 553 (2004)] arXiv:hep-ph/0203079]. 


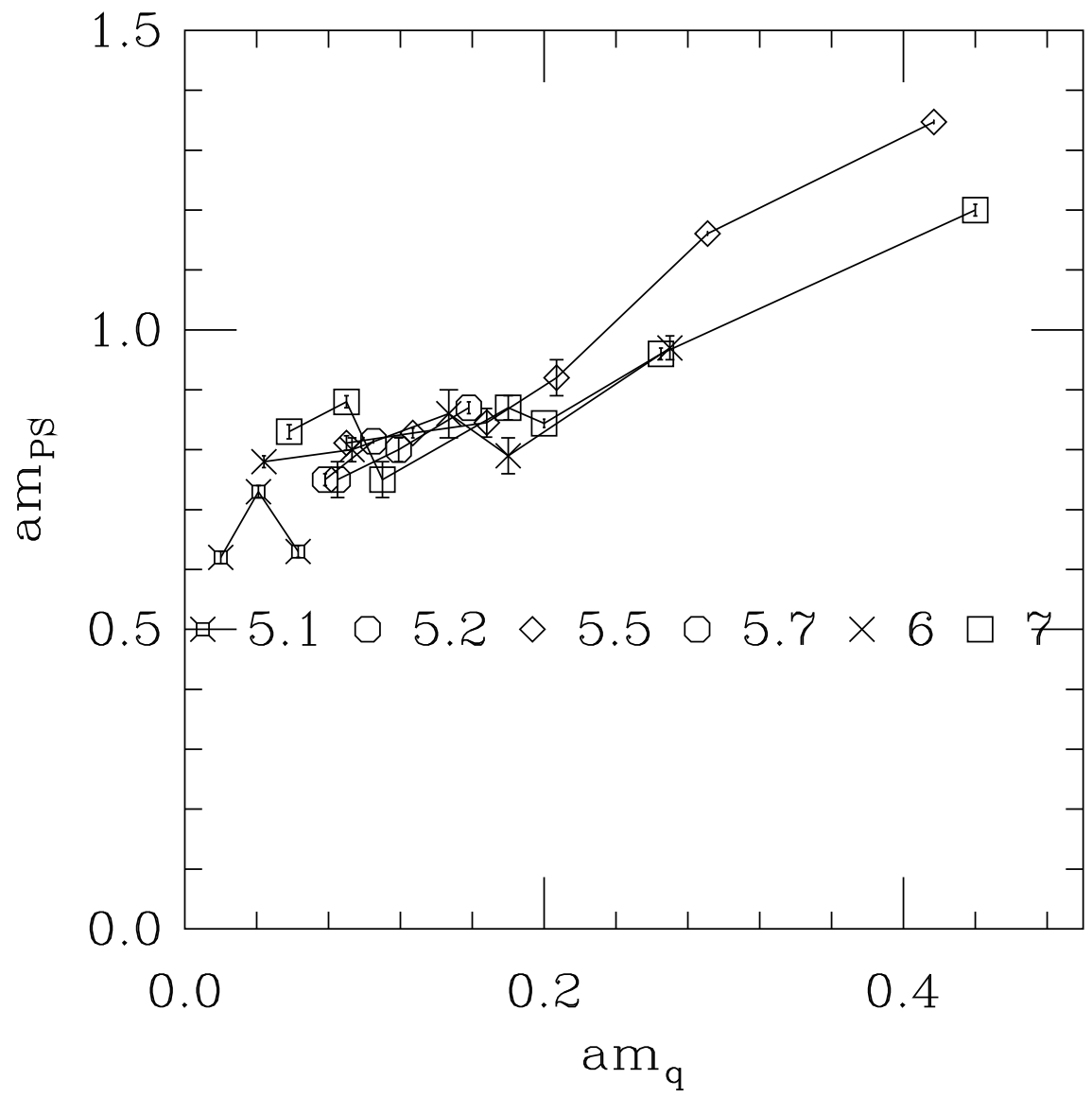

FIG. 2: Replottted data from Ref. [26] for the pseudoscalar screening mass on $\left(12 \times 8^{2}\right) \times 8$ lattices, in the weak coupling phase of $S U(3)$ gauge theory with two flavors of sextet representation quarks. Lines join the equal gauge coupling data, with different plotting symbols for the different $\beta$ values.

[2] H. Georgi, Phys. Rev. Lett. 98, 221601 (2007) arXiv:hep-ph/0703260; Phys. Lett. B 650, 275 (2007) arXiv:0704.2457 [hep-ph]];

[3] W. E. Caswell, Phys. Rev. Lett. 33, 244 (1974).

[4] T. Banks and A. Zaks, Nucl. Phys. B 196, 189 (1982).

[5] T. Appelquist, G. T. Fleming and E. T. Neil, Phys. Rev. Lett. 100, 171607 (2008) arXiv:0712.0609 [hep-ph]].

[6] T. Appelquist, G. T. Fleming and E. T. Neil, arXiv:0901.3766 [hep-ph].

[7] Y. Shamir, B. Svetitsky and T. DeGrand, Phys. Rev. D 78, 031502 (2008) arXiv:0803.1707 [hep-lat]].

[8] A. J. Hietanen, K. Rummukainen and K. Tuominen, arXiv:0904.0864 [hep-lat].

[9] A. G. Cohen and H. Georgi, Nucl. Phys. B 314, 7 (1989).

[10] T. Appelquist, J. Terning and L. C. R. Wijewardhana, Phys. Rev. Lett. 77, 1214 (1996) arXiv:hep-ph/9602385.

[11] T. Appelquist, A. Ratnaweera, J. Terning and L. C. R. Wijewardhana, Phys. Rev. D 58, 105017 (1998) arXiv:hep-ph/9806472.

[12] E. Gardi and G. Grunberg, JHEP 9903, 024 (1999) arXiv:hep-th/9810192.

[13] D. B. Kaplan, J. W. Lee, D. T. Son and M. A. Stephanov, arXiv:0905.4752 [hep-th]. 
[14] A. Deuzeman, M. P. Lombardo and E. Pallante, arXiv:0804.2905 [hep-lat]. X. Y. Jin and R. D. Mawhinney, PoS LATTICE2008, 059 (2008) arXiv:0812.0413 [hep-lat]].

[15] A. Casher, Phys. Lett. B 83, 395 (1979).

[16] J. Greensite and J. Primack, Nucl. Phys. B 180, 170 (1981).

[17] N. Kawamoto and J. Smit, Nucl. Phys. B 192, 100 (1981).

[18] U. M. Heller, Nucl. Phys. Proc. Suppl. 63, 248 (1998) arXiv:hep-lat/9709159.

[19] P. H. Damgaard, U. M. Heller, A. Krasnitz and P. Olesen, Phys. Lett. B 400, 169 (1997) arXiv:hep-lat/9701008.

[20] Y. Iwasaki, K. Kanaya, S. Kaya, S. Sakai and T. Yoshie, Phys. Rev. D 69, 014507 (2004) arXiv:hep-lat/0309159].

[21] G. Mack, Commun. Math. Phys. 53, 155 (1977).

[22] A. Hasenfratz, Phys. Rev. D 80, 034505 (2009) arXiv:0907.0919 [hep-lat].

[23] B. Grinstein, K. A. Intriligator and I. Z. Rothstein, Phys. Lett. B 662, 367 (2008) arXiv:0801.1140 [hep-ph]].

[24] S. Catterall, J. Giedt, F. Sannino and J. Schneible, JHEP 0811, 009 (2008) arXiv:0807.0792 [hep-lat]].

[25] A. J. Hietanen, J. Rantaharju, K. Rummukainen and K. Tuominen, arXiv:0812.1467 [hep-lat].

[26] T. DeGrand, Y. Shamir and B. Svetitsky, Phys. Rev. D 79, 034501 (2009) arXiv:0812.1427 [hep-lat]]. 